\title{
The miRNA-kallikrein axis of interaction: a new dimension in the pathogenesis of prostate cancer
}

\author{
Nicole M.A. White ${ }^{1,2}$, Youssef M. Youssef ${ }^{1}$, \\ Annika Fendler ${ }^{3-5}$, Carsten Stephan ${ }^{3,4}$, Klaus Jung ${ }^{3,4}$ \\ and George M. Yousef ${ }^{1, *}$ \\ ${ }^{1}$ Department of Laboratory Medicine and the Keenan \\ Research Centre in the Li Ka Shing Knowledge Institute, \\ St. Michael's Hospital, Toronto M5B 1W8, Ontario, \\ Canada \\ ${ }^{2}$ Department of Laboratory Medicine and Pathobiology, \\ University of Toronto, Toronto M5S 1A8, Ontario, Canada \\ ${ }^{3}$ Department of Urology, Charité-University Hospital, \\ Charitéplatz, 10117 Berlin, Germany \\ ${ }^{4}$ Berlin Institute for Urologic Research, 10117 Berlin, Germany \\ ${ }^{5}$ Max Delbrück Center for Molecular Medicine, \\ 13125 Berlin-Buch, Germany \\ *Corresponding author \\ e-mail: yousefg@smh.ca
}

\begin{abstract}
Kallikrein-related peptidases (KLKs) are a family of serine proteases that were shown to be useful cancer biomarkers. KLKs have been shown to be dysregulated in prostate cancer (PCa). microRNAs (miRNAs) are short RNA nucleotides that negatively regulate gene expression and have been reportedly dysregulated in PCa. We compiled a comprehensive list of 55 miRNAs that are differentially expressed in PCa from previous microarray analysis and published literature. Target prediction analyses showed that 29 of these miRNAs are predicted to target $10 \mathrm{KLKs}$. Eight of these miRNAs were predicted to target more than one KLK. Quantitative real-time (qRT)-PCR demonstrated that there was an inverse correlation pattern in the expression (normal vs. cancer) between dysregulated miRNAs and their target KLKs. In addition, we experientially validated the miRNA-KLK interaction by transfecting miR-331-3p and miR-143 into a PCa cell line. Decreased expression of targets KLK4 and KLK10, respectively, and decreased cellular growth were observed. In addition to KLKs, dysregulated miRNAs were predicted to target other genes involved in the pathogenesis of PCa. These data show that miRNAs can contribute to KLK regulation in $\mathrm{PCa}$. The miRNA-KLK axis of interaction projects a new element in the pathogenesis of PCa that may have therapeutic implications.
\end{abstract}

Keywords: cancer pathogenesis; kallikrein; KLK; miRNA; personalized medicine; prostate; prostate cancer; tumor markers.

\section{Introduction}

Prostate cancer (PCa) is the most common malignancy in the western world. In 2011, it is predicted that there will be over 240000 newly diagnosed cases of PCa in the United States (American Cancer Society, 2011). The risk of developing PCa increases with age and is highest after the age of 60 . The incidence rate of $\mathrm{PCa}$ has been on the rise over the past three decades, likely due to the recent advances in early detection of the disease. The mechanisms underlying prostate carcinogenesis is yet to be fully elucidated (Gu and Brothman, 2011).

Kallikrein-related peptidases (KLKs) are family of serine proteases well documented in the literature to be dysregulated in a number of cancers including ovarian, prostate, brain, and kidney cancers (Yousef et al., 2001, 2003). Many members of the KLK family have been reported to be dysregulated in $\mathrm{PCa}$ and thus are being investigated as possible biomarkers for the disease (Emami and Diamandis, 2008; Seiz et al., 2010).

Currently, prostate-specific antigen (PSA), which is encoded by the human KLK3 gene is the most widely adopted biomarker for early detection of PCa. However, accumulating evidence suggests the lack of sensitivity and specificity of PSA as a PCa tumor marker. Recent studies have shown that human KLK2 is upregulated during PCa progression and therefore may have use as a biomarker. Diagnostic tests that combine serum KLK2 with total PSA (Recker et al., 2000) and free PSA (Stephan et al., 2006) have improved the sensitivity and specificity of detection. In addition, KLK4 mRNA was shown to be upregulated in PCa tissues (Yousef et al., 1999; Obiezu et al., 2002; Xi et al., 2004; Avgeris et al., 2011) and may play a role in epithelial to mesenchymal transition (Veveris-Lowe et al., 2005). On the other hand, studies showed that KLK10, a potential tumor suppressor, was downregulated in more aggressive PCa s (Luo and Diamandis, 2000). In a PCa cell line, KLK10 mRNA and protein downregulation were suggested to be a result of $\mathrm{CpG}$ island hypermethylation (Sidiropoulos et al., 2005).

microRNAs (miRNAs) are small nonprotein-coding RNA nucleotides that have been widely described as having important biological and regulatory roles in cancer (Garofalo and Croce, 2011). miRNAs negatively regulate the expression of their target genes through complementary, sequence-specific binding, which leads to negative regulation of the target gene by either mRNA degradation or suppression of protein translation. miRNAs have been shown to be dysregulated in 
a wide array of tumors including breast, lung, prostate, ovarian, and kidney cancers (Fendler et al., 2011; White et al., 2011), and their role in the pathogenesis of these cancers is being investigated (Schaefer et al., 2010b; White and Yousef, 2010).

The interaction between miRNAs and KLKs is emerging in the literature as a potential new dimension of regulation of carcinogenesis in many tumors including ovarian and kidney cancers (Chow et al., 2008; Yousef, 2008; White et al., 2010a,b). Recognizing this relationship will enhance our understanding of the mechanisms that control KLK dysregulation in $\mathrm{PCa}$ and will also shed the light on the downstream effector pathways through which miRNAs can affect tumor formation and progression.

In this study, we aimed to examine miRNA dysregulation in PCa and how these dysregulated miRNAs may contribute to KLK dysregulation. We performed target prediction analysis to determine potential KLK targets. We also correlated differential expression of miRNAs dysregulated in PCa with their target KLK expression using quantitative real-time (qRT)-PCR in PCa tissues. We experimentally verified the miRNA-KLK interaction in a PCa cell line model and examined the effect of this interaction on cellular growth. We also performed bioinformatics analyses to help understand the mechanism of miRNA dysregulation in $\mathrm{PCa}$ and how they affect KLKs.

\section{Results}

miRNAs dysregulated in PCa can target KLKs

A compiled total of 55 miRNAs were reported to be dysregulated in PCa by microarray analysis (Schaefer et al., 2010a) and multiple literature sources (Table 1). Twenty-three miRNAs were reported to be upregulated, while 25 were reported to be downregulated. There were conflicting reports for the dysregulation of seven miRNAs (i.e., reported to be upregulated and downregulated in different studies). We performed target prediction analysis on each of the 55 miRNAs and found that $29(53 \%)$ of them are predicted to target KLKs (Table 1). Interestingly, eight of these miRNAs were predicted to target more than one KLK gene; e.g., miR-143 is predicted to target KLK2, KLK5, KLK10, and KLK13. Also, the same KLK can be targeted by more than one miRNA; e.g., KLK10 is predicted to be targeted by eight miRNAs including miR-1, miR-143, and miR-17-5p. The complexity of these potential interactions is shown in Figure 1. Three miRNAs, miR-24, miR-21, and miR-16 were predicted to target both KLK10 and KLK2.

\section{KLKs and miRNAs show inverse correlation pattern of expression in PCa tissues}

In order to validate the miRNA-KLK target interaction in vivo, we validated the expressions of miR-21, miR-24,

Table 1 A complied list of 55 miRNAs that are dysregulated in PCa. Twenty-nine miRNAs are predicted to target KLKs.

\begin{tabular}{|c|c|c|c|c|c|}
\hline miRNA & Expression in $\mathrm{PCa}$ & KLK predicted target & miRNA & Expression in $\mathrm{PCa}$ & KLK predicted target \\
\hline let-7a & Down & - & miR-21 & UP & KLK2, KLK10 \\
\hline let-7i & Down & - & $\operatorname{miR}-221$ & Up/Down & - \\
\hline miR-1 & Up/Down & KLK10, KLK13 & miR-222 & Up/Down & - \\
\hline miR-101 & Down & - & miR-24 & Up & KLK2, KLK3, KLK7, KLK10 \\
\hline miR-103 & Up & KLK5 & miR-30d & Down & - \\
\hline miR-106b & Up/down & - & $\operatorname{miR}-31$ & Up & KLK10 \\
\hline miR-107 & Up/down & KLK5 & miR-32 & $\mathrm{Up}$ & - \\
\hline $\operatorname{miR}-125 b$ & Up & - & $\operatorname{miR}-320$ & Up & - \\
\hline $\operatorname{miR}-126$ & Down & - & miR-324-3p & Down & KLK2 \\
\hline miR-1296 & Down & - & miR-330-3p & Down & KLK4 \\
\hline miR-133a & Up & - & $\operatorname{miR}-331-3 p$ & Down & KLK2, KLK4 \\
\hline miR-133b & Down & - & miR-34a & Down & KLK13 \\
\hline $\operatorname{miR}-140$ & Down & KLK6, KLK9 & $\operatorname{miR}-34 \mathrm{c}$ & Down & KLK13 \\
\hline miR-141 & Up & - & $\operatorname{miR}-363$ & Down & - \\
\hline $\operatorname{miR}-143$ & Down & KLK2, KLK5, KLK10, KLK13 & $\operatorname{miR}-370$ & Down & KLK2 \\
\hline miR-145 & Down & KLK7 & miR-449 & Down & - \\
\hline miR-146a & Down & - & $\operatorname{miR}-449 \mathrm{a}$ & Down & KLK13 \\
\hline $\operatorname{miR}-148 \mathrm{a}$ & Up/Down & - & $\operatorname{miR}-485-3 p$ & Up & - \\
\hline $\operatorname{miR}-15$ & Down & KLK2 & miR-486-5p & Up & KLK11 \\
\hline miR-16 & Up/Down & KLK2, KLK10 & miR-521 & $\mathrm{Up}$ & - \\
\hline $\operatorname{miR}-17-5 p$ & Down & KLK5, KLK7, KLK10 & miR-7 & Down & KLK3 \\
\hline miR-193b & $\mathrm{Up}$ & KLK10 & miR-766 & Up & KLK3 \\
\hline miR-194 & Up & - & $\operatorname{miR}-768-3 p$ & Up & KLK11 \\
\hline miR-197 & $\mathrm{Up}$ & - & miR-801 & Down & - \\
\hline miR-200a & Up & KLK13 & miR-92 & Up & - \\
\hline miR-200b & $\mathrm{Up}$ & KLK7 & miR-96 & $\mathrm{Up}$ & - \\
\hline miR-205 & Down & KLK2 & miR-99 & Down & - \\
\hline miR-20a & $\mathrm{Up}$ & - & & & \\
\hline
\end{tabular}




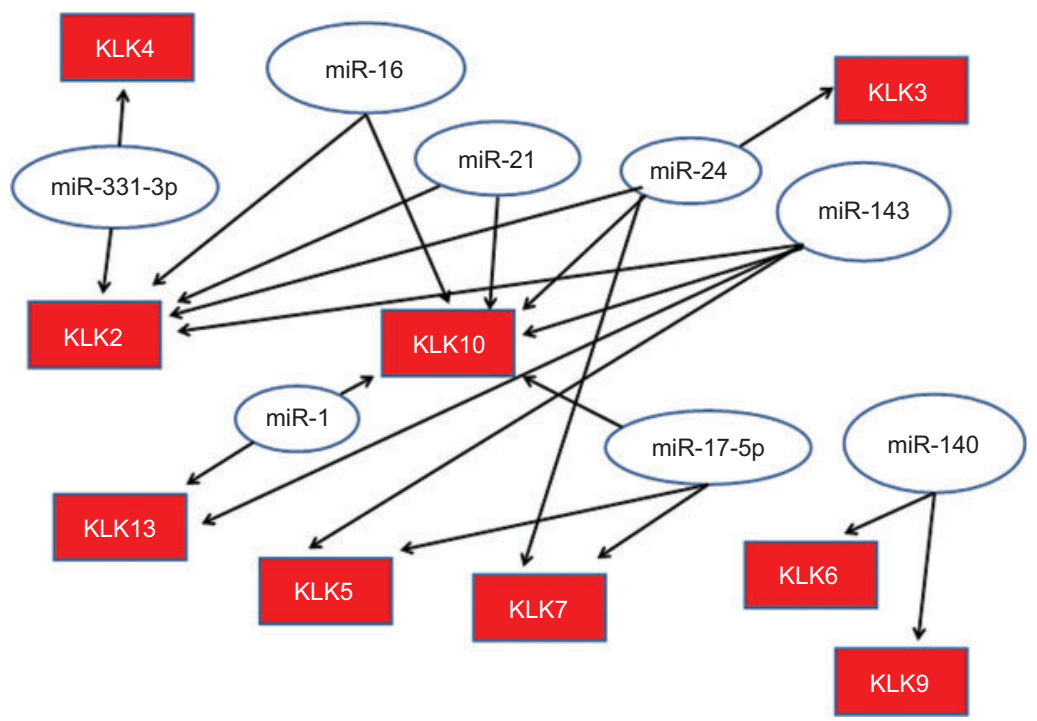

Figure 1 Dysregulated miRNAs in PCa are predicted to target more kallikreins.

The complex interaction between miRNAs and KLKs is shown in the interaction map. Only miRNAs that are predicted to target more than one KLK are shown. Two interesting phenomena are observed; one miRNA can target multiple KLKs, and the same KLK can be targeted by multiple miRNAs.

miR-143, and miR-331-3p and their targeting KLKs in PCa tissues and matched normal tissue from the same patients. We chose these miRNAs because (1) they were significantly dysregulated in $\mathrm{PCa}$ when compared to normal prostate tissue from the same patient; (2) target prediction analysis showed that these miRNAs could target KLKs that have been previously shown to be dysregulated in PCa; and (3) these miRNAs have been previously shown to be dysregulated in cancer.

When we measured the expression of miR-143 and its target KLK2 in 12 pairs of PCa and normal matched prostate tissue from the same patient, we found that $67 \%(8 / 12)$ patients showed increased expression of KLK2. Interestingly, when we examined the relationship between the expression of KLK2 and its targeting miRNA, miR-143, we identified an inverse correlation pattern of expression (Figure 2A and Supplementary Table 1), i.e., when there was decreased levels of miR-143 in cancer compared to normal, there was increased levels of KLK2 expression and vice versa. We also identified other miRNAs that are downregulated in PCa and are predicted to target KLK2. They include miR-15, miR-205, miR324-3p, miR-370, and miR-331-3p. miR-143 (Clape et al., 2009; Peng et al., 2011) and miR-15 (Musumeci et al., 2011) have been shown by others to be downregulated in PCa.

KLK4 was upregulated in 58\% (7/12) PCa cases studied (data not shown) and is predicted to be targeted by two miRNAs, miR330-3p and miR-331-3p, that were downregulated in PCa by us and others (Epis et al., 2009; Lee et al., 2009a). We correlated the expression of miR-331-3p and its target KLK4 and found that there was an inverse correlation pattern of expression (Figure 2B and Supplementary Table 1), suggesting that dysregulated miRNAs can contribute to KLK dysregulation in PCa.

PSA, which was upregulated in 67\% (8/12) PCa tissues (data not shown) examined, is predicted to be a putative target of miR-7, which was downregulated in PCa. Another study showed that miR-7 was downregulated in PCa and can target epidermal growth factor receptor (ERBB) (Giles et al., 2011). KLK3 was also predicted to be targeted by miR-24 and showed an inverse correlation pattern of expression (Supplementary Table 1).

KLK10, which was shown to be downregulated in $75 \%$ (9/12) PCa tissues (data not shown) when compared to normal matched prostate tissue, is predicted to be targeted by five miRNAs that are reported to be upregulated in PCa. These include miR-16, miR-193b, miR-21, miR-24, and miR-31. A number of studies have shown that miR-21 is upregulated and can effect cellular invasion and apoptosis in PCa cell lines (Li et al., 2009b; Ribas and Lupold, 2010). We found that the expression of miR-21 and its target KLK10 were inversely correlated (Supplementary Table 1).

\section{miRNAs can target KLKs and affect cellular growth in a PCa cell line}

In order to experimentally validate the miRNA-KLK interaction, we chose the DU-145 PCa cell line. This cell line model proved to be suitable as it has moderate expression of KLK4 and KLK10 and no expression of miR-331-3p or miR-143, respectively. This is ideal for validation experiments as we would expect to see decreased KLK expression after transfection with its targeting miRNA.

We first transfected DU-145 cells with miR-331-3p and compared the expression of its target, KLK4, before and after transfection (Figure 3A). We found that after transfection with miR-331-3p, there was decreased expression of KLK4 when compared to untransfected cells and control cells $(p<0.001)$. We also experimentally validated the 

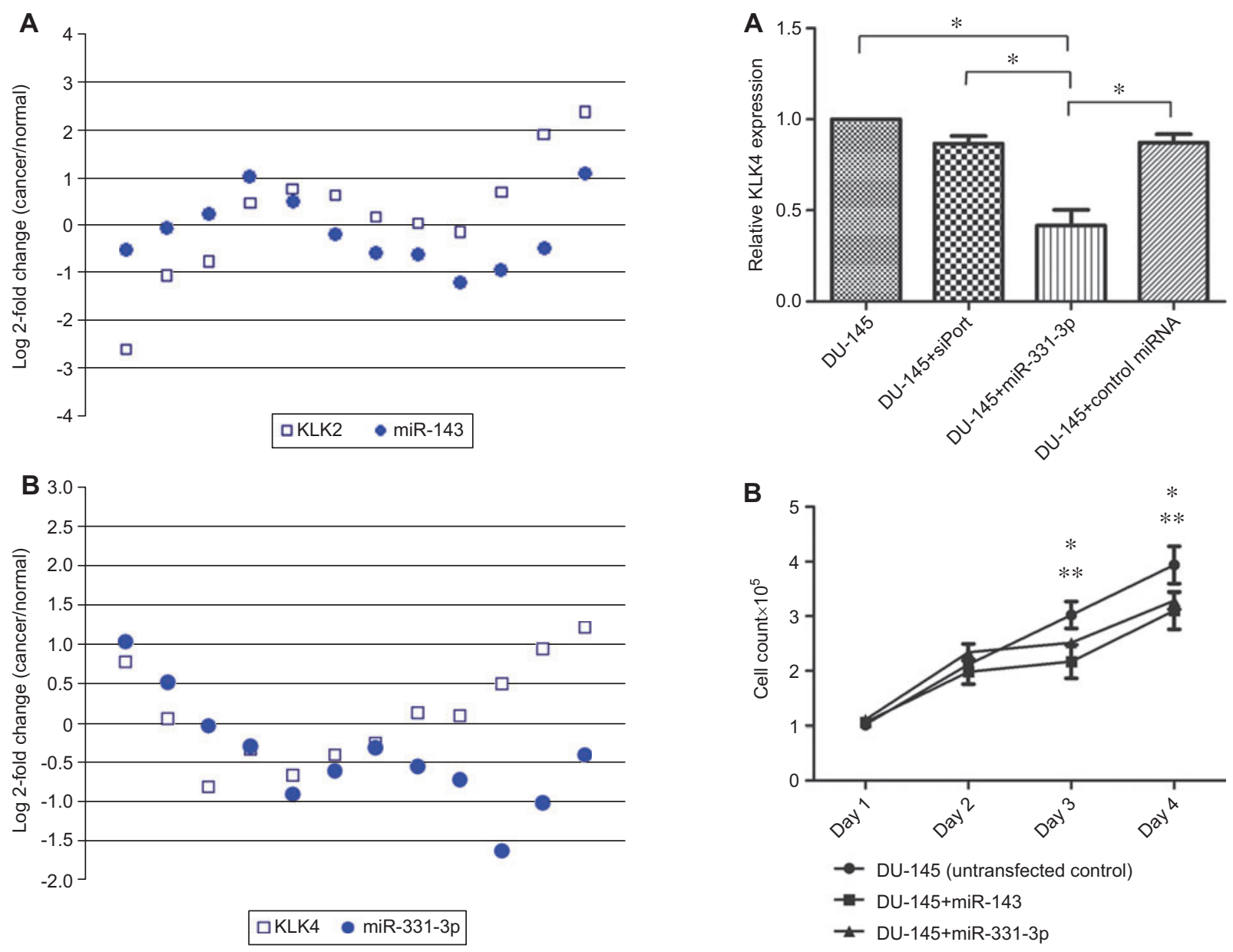

Figure 2 The inverse correlation relationship between miRNAs dysregulated in PCa and their target KLKs.

Expression changes (cancer/normal) were measured for both miRNAs and their target KLKs in pairs of cancer/normal tissues form the same patient. (A) Expression changes of miR-143 (cancer/ normal) are inversely correlated to KLK2 expression in PCa tissues. (B) The expression changes of miR-331-3p are inversely correlated to KLK4 expression.

miR-143 and KLK10 interaction and found that after transfection with miR-143, KLK10 expression was decreased $(p<0.005$; data not shown).

In order to examine the biological effects of the miRNAKLK interaction in PCa, we transfected DU-145 cells with either miR-143 or miR-331-3p and measured their effect on tumor cell growth. We found that cells transfected with either miR-143 $(p<0.001)$ or miR-331-3p $(p<0.05)$ showed a decrease in cellular growth when compared to untransfected cells after days 3 and 4 (Figure 3B), suggesting that the miRNA-KLK interaction can play a role in PCa pathogenesis.

\section{Bioinformatics analysis}

Identification of miRNA clusters located near the KLK cytogenetic locus Previous reports showed that approximately $50 \%$ of human miRNAs are organized into

Figure 3 The effect of dysregulated miRNAs on kallikreins and cell proliferation in a PCa cell line model.

(A) KLK4 expression was significantly decreased in the DU-145 cell line after transfection with miR-331-3p when compared to untransfected controls and control cells. (B) After transfection with either miR-143 or miR-331-3p, cellular growth was decreased in the DU-145 cell line model when compared to untransfected controls after days 3 and $4(* p<0.001$, ** $p<0.05)$.

clusters within large polycistronic messages (Zhang et al., 2009). Using an inter-miRNA cutoff of a distance of 10000 nucleotides, we identified four miRNA clusters that are located around the KLK locus (Table 2). The first cluster has two miRNAs, the second contains three, while the third cluster has 46 miRNAs (which is the largest human miRNA cluster), and the fourth has three miRNAs. Target prediction analysis determined that $72 \%(39 / 54)$ of miRNAs in the clusters were predicted to target KLKs. In order to confirm positive miRNA-KLK interactions, these targets need to be experimentally verified.

Correlation of miRNA with cytogenetic changes in PCa In order to explore the mechanism of miRNA dysregulation in $\mathrm{PCa}$, we compiled a list of frequent chromosomal aberrations in $\mathrm{PCa}$ and correlated them with miRNA dysregulation in the malignancy. We found that there were 22 miRNAs that were reported to be dysregulated in $\mathrm{PCa}$ 
Table 2 Many of the miRNAs that are located in the four clusters around the kallikrein locus are predicted to target KLK.

\begin{tabular}{|c|c|c|c|c|}
\hline Cluster & miRNA & Cytoband & Inter-miRNA distance (nt) & Predicted KLK targets ${ }^{\mathrm{a}}$ \\
\hline \multirow[t]{3}{*}{1} & hsa-miR-330 & $19 \mathrm{q} 13.31$ & - & KLK2, KLK3, KLK4, KLK5, KLK9, KLK10 \\
\hline & hsa-miR-642 & $19 \mathrm{q} 13.31$ & 35841 & KLK2, KLK3, KLK10 \\
\hline & & & Kallikrein locus & \\
\hline \multirow[t]{3}{*}{2} & hsa-miR-99b & $19 \mathrm{q} 13.32$ & - & \\
\hline & hsa-let-7e & $19 \mathrm{q} 13.32$ & 105 & KLK2, KLK3, KLK6, KLK10, KLK15 \\
\hline & hsa-miR-125a & $19 \mathrm{q} 13.32$ & 390 & KLK2, KLK5, KLK6, KLK10, KLK5 \\
\hline \multirow[t]{46}{*}{3} & hsa-miR-512-1 & $19 \mathrm{q} 13.33$ & - & KLK2, KLK3, KLK7, KLK10 \\
\hline & hsa-miR-512-2 & $19 \mathrm{q} 13.33$ & 2395 & KLK2, KLK3, KLK7, KLK10 \\
\hline & hsa-miR-1323 & $19 \mathrm{q} 13.33$ & 2714 & \\
\hline & hsa-miR-498 & $19 \mathrm{q} 13.33$ & 2157 & KLK11 \\
\hline & hsa-miR-520e & $19 \mathrm{q} 13.33$ & 1391 & KLK2, KLK10 \\
\hline & hsa-miR-515-1 & $19 \mathrm{q} 13.33$ & 3206 & KLK2, KLK3, KLK7, KLK10, KLK13 \\
\hline & hsa-miR-519e & $19 \mathrm{q} 13.33$ & 855 & KLK2, KLK3, KLK5, KLK10, KLK13 \\
\hline & hsa-miR-520f & $19 \mathrm{q} 13.33$ & 2136 & KLK3 \\
\hline & hsa-miR-515-2 & $19 \mathrm{q} 13.33$ & 2764 & KLK2, KLK3, KLK7, KLK10, KLK13 \\
\hline & hsa-miR-519c & $19 \mathrm{q} 13.33$ & 1378 & KLK7 \\
\hline & hsa-miR-1283-1 & $19 q 13.33$ & 1926 & \\
\hline & hsa-miR-520a & $19 q 13.33$ & 2314 & KLK2, KLK3, KLK7, KLK10 \\
\hline & hsa-miR-526b & $19 \mathrm{q} 13.33$ & 3428 & KLK7, KLK10, KLK15 \\
\hline & hsa-miR-519b & $19 \mathrm{q} 13.33$ & 738 & KLK7 \\
\hline & hsa-miR-525 & $19 \mathrm{q} 13.33$ & 2240 & KLK2, KLK6, KLK9 \\
\hline & hsa-miR-523 & $19 \mathrm{q} 13.33$ & 768 & \\
\hline & hsa-miR-518f & $19 \mathrm{q} 13.33$ & 1544 & \\
\hline & hsa-miR-520b & $19 \mathrm{q} 13.33$ & 1126 & KLK10 \\
\hline & hsa-miR-518b & $19 \mathrm{q} 13.33$ & 1450 & \\
\hline & hsa-miR-526a-1 & $19 \mathrm{q} 13.33$ & 3433 & KLK9 \\
\hline & hsa-miR-520c & $19 \mathrm{q} 13.33$ & 1117 & KLK9, KLK10 \\
\hline & hsa-miR-518c & $19 \mathrm{q} 13.33$ & 1196 & \\
\hline & hsa-miR-524 & $19 q 13.33$ & 2167 & \\
\hline & hsa-miR-517a & $19 \mathrm{q} 13.33$ & 1180 & KLK2 \\
\hline & hsa-miR-519d & $19 \mathrm{q} 13.33$ & 993 & KLK5, KLK7, KLK10 \\
\hline & hsa-miR-521-2 & $19 \mathrm{q} 13.33$ & 3160 & \\
\hline & hsa-miR-520d & $19 \mathrm{q} 13.33$ & 3416 & KLK2, KLK10 \\
\hline & hsa-miR-517b & $19 \mathrm{q} 13.33$ & 894 & \\
\hline & hsa-miR-520g & $19 \mathrm{q} 13.33$ & 1024 & KLK10 \\
\hline & hsa-miR-516b-2 & $19 \mathrm{q} 13.33$ & 3187 & KLK2, KLK3, KLK10 \\
\hline & hsa-miR-526a-2 & $19 \mathrm{q} 13.33$ & 1396 & KLK9 \\
\hline & hsa-miR-518e & $19 \mathrm{q} 13.33$ & 2852 & \\
\hline & hsa-miR-518a-1 & $19 \mathrm{q} 13.33$ & 1081 & \\
\hline & hsa-miR-518d & $19 \mathrm{q} 13.33$ & 3787 & KLK2 \\
\hline & hsa-miR-516b-1 & $19 \mathrm{q} 13.33$ & 1882 & KLK2, KLK3, KLK10 \\
\hline & hsa-miR-518a-2 & $19 \mathrm{q} 13.33$ & 2399 & \\
\hline & hsa-miR-517c & $19 q 13.33$ & 1894 & KLK2 \\
\hline & hsa-miR-520h & $19 \mathrm{q} 13.33$ & 1105 & KLK10 \\
\hline & hsa-miR-521-1 & $19 \mathrm{q} 13.33$ & 6037 & \\
\hline & hsa-miR-522 & $19 q 13.33$ & 2489 & KLK3, KLK10 \\
\hline & hsa-miR-519a-1 & $19 \mathrm{q} 13.33$ & 1100 & KLK7 \\
\hline & hsa-miR-527 & $19 \mathrm{q} 13.33$ & 1537 & KLK12 \\
\hline & hsa-miR-516a-1 & $19 \mathrm{q} 13.33$ & 2639 & KLK9 \\
\hline & hsa-miR-1283-2 & $19 \mathrm{q} 13.33$ & 1402 & \\
\hline & hsa-miR-516a-2 & $19 \mathrm{q} 13.33$ & 2815 & KLK9 \\
\hline & hsa-miR-519a-2 & $19 \mathrm{q} 13.33$ & 1122 & KLK7 \\
\hline \multirow{3}{*}{4} & hsa-miR-371 & $19 \mathrm{q} 13.33$ & - & KLK2, KLK10 \\
\hline & hsa-miR-372 & $19 \mathrm{q} 13.33$ & 149 & KLK5, KLK10 \\
\hline & hsa-miR-373 & $19 \mathrm{q} 13.33$ & 749 & KLK2, KLK5, KLK10 \\
\hline
\end{tabular}

${ }^{a}$ KLKs were only listed if they were predicted to be targeted by at least three programs.

that were located at sites of reported chromosomal aberrations in PCa. Fifty-nine percent (13/22) miRNAs were reported to be correlated with the direction of chromosomal change in $\mathrm{PCa}$; e.g., miR-449 and miR-449a are downregulated in PCa and located at $\mathrm{Ch} 5 \mathrm{q} 11.2$, which has been reported to have chromosomal losses in PCa (Table 3). These data suggest that chromosomal changes may, in part, contribute to miRNA dysregulation in $\mathrm{PCa}$. 
Table 3 miRNAs that are dysregulated in PCa are located at sites of frequently reported chromosomal aberrations.

\begin{tabular}{lccc}
\hline miRNA & $\begin{array}{c}\text { miRNA } \\
\text { dysregulation }\end{array}$ & $\begin{array}{c}\text { Chromosomal } \\
\text { location }\end{array}$ & $\begin{array}{c}\text { Reported } \\
\text { chromosomal } \\
\text { changes }\end{array}$ \\
\hline miR-1 & Up & $20 \mathrm{q} 13.33$ & Gain \\
miR-106b & Up & $7 \mathrm{q} 22.1$ & Gain \\
miR-24 & Up & $9 \mathrm{q} 22.32$ & Gain \\
miR-30d & Down & $8 \mathrm{q} 24.22$ & Gain \\
miR-32 & Up & $9 \mathrm{q} 31.3$ & Gain \\
miR-96 & Up & $7 \mathrm{q} 32.2$ & Gain \\
miR-107 & Down & $10 \mathrm{q} 23.31$ & Loss \\
miR1296 & Down & $10 \mathrm{q} 21.3$ & Loss \\
miR-140 & Down & $16 \mathrm{q} 22.1$ & Loss \\
miR-15 & Down & $13 \mathrm{q} 14.2$ & Loss \\
miR-324-3p & Down & $17 \mathrm{p} 13.1$ & Loss \\
miR-449 & Down & $5 \mathrm{q} 11.2$ & Loss \\
miR-449a & Down & $5 \mathrm{q} 11.2$ & Loss \\
\hline
\end{tabular}

Involvement of dysregulated miRNAs in the pathogenesis of PCa To examine the role of miRNAs that are dysregulated in PCa and can target KLKs in PCa pathogenesis, we did target prediction analysis for these 29 miRNAs and examined their potential interaction with pathways that are frequently dysregulated in PCa (Table 4). We found that 16 of these miRNAs were validated to target components of pathways frequently dysregulated in $\mathrm{PCa}$. One of these pathways that are hyperactivated in approximately 30\%$50 \%$ of PCas is the phophatidylinositol 3-kinase/RAC-alpha serine/theronine-protein kinase (PI3K/Akt) signaling pathway (Sarkar et al., 2010). A major component of this pathway is the tumor suppressor phosphatase and tensin homolog (PTEN), which normally functions to block the activity of $\mathrm{PI} 3 \mathrm{~K}$. Another pathway that is frequently dysregulated in PCa includes the androgen receptor (AR) pathway.

miR-331-3p, which is decreased in PCa and is predicted to target KLK2 and KLK4, has been shown to target two specific sites within the human ERBB2 mRNA 3' untranslated region. Transfection of multiple PCa cell lines with miR-331 showed a reduction of ERBB2 mRNA and protein expression and a block of the downstream PI3K/Akt signaling pathway. miR-331-3p was also shown to block androgen signaling and reduce the activity of the PSA promoter, subsequently decreasing PSA expression (Epis et al., 2009).

miR-21, which is described as an oncomir, has been shown to have increased expression in almost all cancers including PCa. Validated targets for miR-21 in PCa include bone morphogenetic protein receptor, type II (BMPRII), myristoylated alanine-rich protein kinase $\mathrm{C}$ substrate (MARCKS), programmed cell death 4 (PDCD4), PTEN, acidic (leucine-rich) nuclear phosphoprotein 32 family, member A (ANP32A), SWItch/Sucrose NonFermentable (SWI/SNF) related, matrix-associated, actin-dependent regulator of chromatin, subfamily a, member 4 (SMARCA4), and sprouty 1 (SPRY1; Table 4). Yang et al. showed that when miR-21 was ectopically expressed in PCa cells, phosphorylation of Akt was induced by interferon but not induced in cells that do not express miR-21 (Yang et al., 2010). In addition, it has been shown that miR-21 can target the tumor suppressor PTEN (Liu et al., 2011a). Ribas et al. showed that AR can directly interact with miR-21 regulatory regions, indicating

Table 4 A complied list of 16 miRNAs that are dysregulated in PCa, predicted to target KLKs and have been validated to target other genes involved in prostate carcinogenesis.

\begin{tabular}{|c|c|c|c|}
\hline miRNA & Expression in $\mathrm{PCa}$ & Validated targets & References \\
\hline miR-107 & Up/Down & GRN & (Wang et al., 2010) \\
\hline $\operatorname{miR}-143$ & Down & MYO6, KRAS, ERK5, ICP4 & $\begin{array}{l}\text { (Clape et al., 2009; Lee et al., 2009a; Szczyrba et al., 2010; Xu } \\
\text { et al., 2011) }\end{array}$ \\
\hline $\operatorname{miR}-145$ & Down & $\begin{array}{l}\text { MYO6, ICP4, SWAP70, } \\
\text { FSCN1, TNFSF10 }\end{array}$ & $\begin{array}{l}\text { (Lee et al., 2009a; Szczyrba et al., 2010; Zaman et al., 2010; } \\
\text { Chiyomaru et al., 2011; Fuse et al., 2011) }\end{array}$ \\
\hline miR-15 & Down & BCL2, CCND1, WNT3A & (Bonci et al., 2008) \\
\hline miR-16 & Up & BCL2, CCND1, WNT3A & (Bonci et al., 2008) \\
\hline miR-200b & Up & ZEB2 & (Kong et al., 2009) \\
\hline $\operatorname{miR}-205$ & Down & BCL2 & (Bhatnagar et al., 2010) \\
\hline $\operatorname{miR}-21$ & Up & $\begin{array}{l}\text { BMPRII, MARCKS, } \\
\text { PDCD4, PTEN, ANP32A, } \\
\text { SMARCA4, SPRY1 }\end{array}$ & $\begin{array}{l}\text { (Qin et al., 2009; Finlayson and Freeman, 2009; Shi et al., } \\
\text { 2010; Yang et al., 2010; Darimipourain et al., 2011; Liu et al., } \\
\text { 2011b; Schramedei et al., 2011) }\end{array}$ \\
\hline $\operatorname{miR}-24$ & Up & FAF1 & (Qin et al., 2010) \\
\hline miR-31 & $\mathrm{Up}$ & E2F6 & (Bhatnagar et al., 2010) \\
\hline $\operatorname{miR}-330-3 p$ & Down & $\mathrm{E} 2 \mathrm{~F} 1$ & (Lee et al., 2009b) \\
\hline $\operatorname{miR}-331-3 p$ & Down & ERBB2, PSA & (Epis et al., 2009) \\
\hline $\operatorname{miR}-34 \mathrm{a}$ & Down & CD44, SIRT1, BCL2 & (Fujita et al., 2008; Kojima et al., 2010; Liu et al., 2011a) \\
\hline $\operatorname{miR}-34 \mathrm{c}$ & Down & E2F3, BCL2 & (Hagman et al., 2010) \\
\hline $\operatorname{miR}-449 a$ & Down & HDAC-1, CCND1 & (Noonan et al., 2009; Noonan et al., 2010) \\
\hline $\operatorname{miR}-7$ & Down & EGFR & (Giles et al., 2011) \\
\hline
\end{tabular}


direct transcriptional induction (Ribas et al., 2009). They also found that overexpression of miR-21 enhanced tumor xenograft growth and was sufficient to support androgenindependent proliferation following surgical castration. Taken together, our data shows that KLKs can be involved in a network of interactions that are involved in PCa pathogenesis.

\section{Discussion}

Kallikreins have been shown to be involved in a number of different cancers, yet the mechanisms controlling their dysregulation are still uncertain for many cases. It is hypothesized that, in part, this regulation may be posttranscriptional through miRNAs (Chow et al., 2008; Yousef, 2008). We showed evidence indicating that miRNAs can contribute to KLK dysregulation in PCa through two independent lines of evidence. First, we observed a pattern of inverse correlation between dysregulated miRNAs and their target KLKs, miR-143 and its target KLK2, and miR-331-3p and its target KLK4 in PCa tissue (Figure 2), indicating that the miRNAs may control KLK expression in PCa. In addition, we showed that transfection of miR-143 into a PCa cell line model decreased KLK2 expression and cellular growth. This, however, must be interpreted with caution. It must be noted that miR-143 can have other targets that may contribute to this biological effect. Overexpression of miR-143 has been shown to suppress $\mathrm{PCa}$ proliferation and migration and increase sensitivity to docetaxel by targeting the mitogen-activated protein kinase signaling (EGFR/RAS/MAPK) pathway (Xu et al., 2011). Other targets that have been validated for miR-143 in PCa include extracellular signal-regulated kinase-5 (ERK5) (Clape et al., 2009) and genes involved in epithelial to mesenchymal transition (Peng et al., 2011).

The fact that miRNAs have multiple targets and can target more than one gene has important implications. On one hand, the targeting of a number of genes in the same pathway has potential therapeutic implications. In this case, the introduction of a miRNA can target or 'hit' the pathway at multiple points, which increases the chances of successful pathway suppression (Metias et al., 2009; White and Yousef, 2011). On the other hand, because miRNAs can have a number of targets in different pathways, it is experimentally challenging to control for off-target effects. This needs solid experimental design, combination of bioinformatics and experimental approaches, and the use multiple independent validation techniques.

We preformed bioinformatics analyses to explore the mechanism of miRNA dysregulation in PCa. The coordinated dysregulation of miRNA clusters, miRNAs that are located in close proximity, implies a coordinated regulation and function. Our analysis showed that the KLK locus, Ch 19q13, harbors a large number of miRNAs. This miRNA cluster, which has been termed C19MC, represents the largest cluster in the human genome (Bentwich et al., 2005). Interestingly, an amplicon of this region was found to be associated with an aggressive subgroup of primitive neuroectodermal tumors with distinct gene-expression profiles, characteristic histology, and dismal survival (Li et al., 2009a). Coordinated expression of miRNAs within clusters suggests a coordinated control mechanism and functions. Similarly, it has been suggested that KLKs share a similar control mechanism and function. It would be interesting to explore if a relationship exists between the two.

Other groups have examined the miRNA-KLK interaction in PCa by examining the relationship between miRNAs and PSA (KLK3) in PCa. Transfection of miR-99a, miR-99b, or miR-100 inhibited the growth of PCa cells and decreased the expression of PSA (KLK3) in PCa cell lines (Sun et al., 2011). PSA levels increased after inhibition of the miR-99 family, suggesting that miRNAs can contribute to PSA regulation in $\mathrm{PCa}$.

miRNAs represent attractive useful clinical markers for $\mathrm{PCa}$, and evidence for this use is emerging quickly. Hoa et al. reported an increase in the positive predictive value in $\mathrm{PCa}$ patients from $40 \%$ to $87.5 \%$ by integrating PSA blood levels with miR-21 and miR-141 profiles (Hao et al., 2011). It has also been reported that a panel of markers including 10 protein-coding genes and two miRNAs, miR-519d and miR647, was identified to accurately separate patients with and without biochemical recurrence $(p<0.001)$, as well as for the subset of 42 Gleason score of [FCP1] seven patients $(p<0.001)$ (Long et al., 2011). More recently, circulating miR-141 levels in the plasma of PCa patients predicted clinical outcomes with an odds ratio of at least 8.3 (Gonzales et al., 2011). In the same study, miR-141 also had the highest correlation with temporal changes of PSA and demonstrated high correlation with changes of the other biomarkers. Circulating tumor markers represent attractive clinical markers as their non-invasive nature if superior to prostatectomy.

Through our study and others, it is now evident that the miRNA-KLK interaction is involved in PCa pathogenesis and has potential clinical utilities. However, this relationship is more complex than previously thought. One miRNA can target more than one KLK, and one KLK can be targeted by more than one miRNA leading to a complex network of interactions (Figure 1). In addition, these interactions may be direct or indirect, which adds to the complexity. Although miRNAs act posttranscriptionally, recent evidence supports that they also usually interfere with the stability of their target mRNAs (Cheng and Li, 2008; Gennarino et al., 2009; Guimbellot et al., 2009) Moreover, KLKs may also contribute to miRNA regulation through transcriptional regulation of miRNA promoters as suggested by KLK nuclear localization (Korkmaz et al., 2001; Klokk et al., 2007).

In conclusion, we provide bioinformatics and experimental evidence suggesting that miRNAs are involved in the regulation of KLKs and that both can actively contribute to PCa formation and progression.

\section{Materials and methods}

\section{Sample collection}

The study was approved by the Research Ethics Boards of St. Michael's Hospital, Toronto, Canada, and Charité-University Hospital, Berlin, Germany. Fresh prostate tissues from 12 pairs of tumor and normal tissues from the same patients were sampled directly after 
surgical removal of the gland for treatment of PCa at Charité-University Hospital, Berlin, Germany. One full frontal section, which was grossly tumor suspicious, was deep frozen in liquid nitrogen. A diagnostic hematoxylin and eosin section was prepared to verify tumor content and margin status and to identify areas of normal and tumor tissue. These regions of interest were punch biopsied, and another frozen section was made to ascertain tumor content and to assign a Gleason score. Only cases with $>90 \%$ tumor tissue were considered for further analysis.

\section{miRNA dysregulation in PCa}

In order to create a comprehensive list of miRNAs dysregulated in $\mathrm{PCa}$, we combined data from our recently published miRNA microarray comparing $\mathrm{PCa}$ with normal matched prostate tissue from the same patient (Schaefer et al., 2010a) and did a literature search for additional reported miRNAs dysregulated in PCa when compared to normal prostate tissue (Sylvestre et al., 2007; Ambs et al., 2008; Shi et al., 2011; Sun et al., 2011).

\section{Total RNA extraction and reverse transcription}

Frozen matched malignant and non-malignant samples were collected in RNAlater Stabilization reagent (Qiagen GmbH, Hilden, Germany). RNA was extracted with the RNA miR Isolation Kit (Qiagen). Total RNA yields and A260/280 ratio were monitored with a NanoDrop 2000 spectrophotometer (NanoDrop Technologies, Wilmington, DE, USA), and RNA integrity numbers were measured with the 2100 Bioanalyzer (Agilent Technologies, Santa Clara, CA, USA). Only RNA extracts with RNA integrity number values $>6$ were included in further analysis. Samples were stored at $-80^{\circ} \mathrm{C}$ until further use.

One microgram of total RNA was reverse transcribed using the Transcriptor First Strand cDNA Synthesis Kit (Roche Applied Science, Indianapolis, IN, USA) following the manufacturer's recommendation.

\section{qRT-PCR}

qRT-PCR was performed using the Step One ${ }^{\mathrm{TM}}$ Plus Real-Time PCR System (Applied Biosystems, Foster City, CA, USA). Primer/probe sets were purchased as premade TaqMan Assays on Demand for $K L K 2$ (Hs00428383_m1), KLK3 (Hs02576345_m1), KLK4 (Hs00191772_ $\mathrm{m} 1$ ), and $K L K 10$ (Hs00173611_m1). Thermal cycling conditions were according to the manufacture's fast protocol, and all reactions were performed in triplicate. Relative expression was determined using the $\Delta \Delta \mathrm{C}_{\mathrm{T}}$ method, and expression values were normalized to hypoxanthine phosphoribosyltransferase 1 (HPRT1) (Hs01003267_m1).

\section{miRNA target prediction and validation analysis}

miRNA target prediction analyses were performed using TargetScan 5.1 (Lewis et al., 2005) and miRecords (Xiao et al., 2009). Experimental target validation of miRNA-KLK interactions were assayed by transfecting the PCa cell line DU-145 with hsa-miR-331-3p and hsa-miR-143 (Ambion, Foster City, CA, USA) and comparing the expression levels of target KLKs before and after transfection. Cells were transfected using the si-PORT ${ }^{\mathrm{TM}} \mathrm{NeoFX}{ }^{\mathrm{TM}}$ transfection system (Ambion) according to the manufacturer's protocol. miRNA precursors, hsa-miR-331-3p, hsa-miR-143, and a random sequence control (Applied Biosystems) were added at a final concentration of $5 \mathrm{~nm}$. Transfection agent/RNAmolecule complex formation was carried out in Opti-MEM Reduced-Serum Media (1X; Invitrogen, Burlington, ON, Canada). The DU-145 cell line was maintained in
RPMI 1640 supplemented with 10\% fetal bovine serum (Invitrogen, Burlington, Canada) in $5 \% \mathrm{CO}_{2}$ and $37^{\circ} \mathrm{C}$.

\section{Cell proliferation assay}

Cellular proliferation was measured by cell counting. DU-145 cells were plated at $8.0 \times 10^{4}$ cells per well in a 12 -well plate and were either untransfected or transfected with miR-143, miR-331-3p, or a random sequence control miRNA. Cells were incubated for 1-4 days after which they were trypsinized and counted. Cells were counted in triplicate and repeated thrice.

\section{In silico analysis}

Chromosomal aberrations in $\mathrm{PCa}$ were extracted from the Progenetix database (www.progenetix.net). Average genetic changes from 687 cases of PCa were used for comparative genomic hybridization analysis. We also did a literature search for cytogenetic aberrations in PCa (Nupponen and Visakorpi, 2000; Brothman, 2002; Gu and Brothman, 2011). Results were compiled and were then compared to the chromosomal locations of dysregulated miRNAs in PCa.

miRNA clusters were defined as miRNAs, which lie within 10000 nucleotides (inter-miRNA distance) from one another. The distance between miRNA and their chromosomal locations were determined using the latest release (Release 17) of miRBase and the UCSC's Genome Browser and Track feature.

Sequence homology and multiple alignment tools were performed using miROrtho (Faculty of Medicine, University of Geneva), which contains predictions of miRNA genes covering several animal genomes. Built into miROrtho is a T-COFFEE (version 6.92) output for the multiple sequence alignment. High conservation was defined as sequences having an alignment reliability core index $>80$.

\section{Acknowledgements}

This work was supported by grants from the Canadian Cancer Society (CCS grant \# 20185), the Ministry of Research and Innovation of the Government of Ontario, the Kidney Foundation of Canada, and the Cancer Research Society.

\section{References}

Ambs, S., Prueitt, R.L., Yi, M., Hudson, R.S., Howe, T.M., Petrocca, F., Wallace, T.A., Liu, C.G., Volinia, S., Calin, G.A., et al. (2008). Genomic profiling of microRNA and messenger RNA reveals deregulated microRNA expression in prostate cancer. Cancer Res. 68, 6162-6170.

American Cancer Society (2011). American Cancer Society: Cancer Facts and Figures 2011. (Atlanta, GA).

Avgeris, M., Stravodimos, K., and Scorilas, A. (2011), GA. Kallikrein-related peptidase 4 gene (KLK4) in prostate tumors: quantitative expression analysis and evaluation of its clinical significance. Prostate 71, 1780-1789.

Bentwich, I., Avniel, A., Karov, Y., Aharonov, R., Gilad, S., Barad, O., Barzilai, A., Einat, P., Einav, U., Meiri, E., et al. (2005). Identification of hundreds of conserved and nonconserved human microRNAs. Nat. Genet. 37, 766-770.

Bhatnagar, N., Li, X., Padi, S.K., Zhang, Q., Tang, M.S., and Guo, B. (2010). Downregulation of miR-205 and miR-31 confers resistance to chemotherapy-induced apoptosis in prostate cancer cells. Cell Death. Dis. 1, e105. 
Bonci, D., Coppola, V., Musumeci, M., Addario, A., Giuffrida, R., Memeo, L., D’Urso, L., Pagliuca, A., Biffoni, M., Labbaye, C, et al. (2008). The miR-15a-miR-16-1 cluster controls prostate cancer by targeting multiple oncogenic activities. Nat. Med. 14, 1271-1277.

Brothman, A.R. (2002). Cytogenetics and molecular genetics of cancer of the prostate. Am. J. Med. Genet. 115, 150-156.

Cheng, C. and Li, L.M. (2008). Inferring microRNA activities by combining gene expression with microRNA target prediction. PLoS One 3, e1989.

Chiyomaru, T., Tatarano, S., Kawakami, K., Enokida, H., Yoshino, H., Nohata, N., Fuse, M., Seki, N., and Nakagawa, M. (2011). SWAP70, actin-binding protein, function as an oncogene targeting tumor-suppressive miR-145 in prostate cancer. Prostate 71 , $1559-1567$.

Chow, T.F., Crow, M., Earle, T., El-Said, H., Diamandis, E.P., and Yousef, G.M. (2008). Kallikreins as microRNA targets: an in silico and experimental-based analysis. Biol. Chem. 389, 731738.

Clape, C., Fritz, V., Henriquet, C., Apparailly, F., Fernandez, P.L., Iborra, F., Avances, C., Villalba, M., Culine, S., and Fajas, L. (2009). miR-143 interferes with ERK5 signaling, and abrogates prostate cancer progression in mice. PLoS One 4, e7542.

Darimipourain, M., Wang, S., Ittmann, M., and Kwabi-Addo, B. (2011). Transcriptional and post-transcriptional regulation of Sprouty1, a receptor tyrosine kinase inhibitor in prostate cancer. Prostate Cancer Prostatic Dis. 14, 279-285.

Emami, N. and Diamandis, E.P. (2008). Utility of kallikrein-related peptidases (KLKs) as cancer biomarkers. Clin. Chem. 54, 1600-1607.

Epis, M.R., Giles, K.M., Barker, A., Kendrick, T.S., and Leedman, P.J. (2009). miR-331-3p regulates ERBB-2 expression and androgen receptor signaling in prostate cancer. J. Biol. Chem. 284, 24696-24704.

Fendler, A., Jung, M., Stephan, C., Honey, R.J., Stewart, R.J., Pace, K.T., Erbersdobler, A., Samaan, S., Jung, K., and Yousef, G.M. (2011). miRNAs can predict prostate cancer biochemical relapse and are involved in tumor progression. Int. J. Oncol. 39, $1183-1192$.

Finlayson, A.E. and Freeman, K.W. (2009). A cell motility screen reveals role for MARCKS-related protein in adherens junction formation and tumorigenesis. PLoS One 4, e7833.

Fujita, Y., Kojima, K., Hamada, N., Ohhashi, R., Akao, Y., Nozawa, Y., Deguchi, T., and Ito, M. (2008). Effects of miR-34a on cell growth and chemoresistance in prostate cancer PC3 cells. Biochem. Biophys. Res. Commun. 377, 114-119.

Fuse, M., Nohata, N., Kojima, S., Sakamoto, S., Chiyomaru, T., Kawakami, K., Enokida, H., Nakagawa, M., Naya, Y., Ichikawa, T., et al. (2011). Restoration of miR-145 expression suppresses cell proliferation, migration and invasion in prostate cancer by targeting FSCN1. Int. J. Oncol. 38, 1093-1101.

Garofalo, M. and Croce, C.M. (2011). microRNAs: master regulators as potential therapeutics in cancer. Annu. Rev. Pharmacol. Toxicol. 51, 25-43.

Gennarino, V.A., Sardiello, M., Avellino, R., Meola, N., Maselli, V., Anand, S., Cutillo, L., Ballabio, A., and Banfi, S. (2009). MicroRNA target prediction by expression analysis of host genes. Genome Res. 19, 481-490.

Giles, K.M., Barker, A., Zhang, P.M., Epis, M.R., and Leedman, P.J. (2011) MicroRNA regulation of growth factor receptor signaling in human cancer cells. Methods Mol.Biol. 676, 147-163.

Gonzales, J.C., Fink, L.M., Goodman, O.B. Jr., Symanowski, J.T., Vogelzang, N.J., and Ward, D.C. (2011). Comparison of circulating MicroRNA 141 to circulating tumor cells, lactate dehydrogenase, and prostate-specific antigen for determining treatment response in patients with metastatic prostate cancer. Clin. Genitourin. Cancer 9, 39-45.

Gu, G. and Brothman, A.R. (2011). Cytogenomic aberrations associated with prostate cancer. Cancer Genet. 204, 57-67.

Guimbellot, J.S., Erickson, S.W., Mehta, T., Wen, H., Page, G.P., Sorscher, E.J., and Hong, J.S. (2009). Correlation of microRNA levels during hypoxia with predicted target mRNAs through genome-wide microarray analysis. BMC Med. Genomics 2, 15.

Hagman, Z., Larne, O., Edsjo, A., Bjartell, A., Ehrnstrom, R.A., Ulmert, D., Lilja, H., and Ceder, Y. (2010). miR-34c is downregulated in prostate cancer and exerts tumor suppressive functions. Int. J. Cancer 127, 2768-2776.

Hao, Y., Zhao, Y., Zhao, X., He, C., Pang, X., Wu, T.C., Califano, J.A., and Gu, X. (2011). Improvement of prostate cancer detection by integrating the PSA test with miRNA expression profiling. Cancer Invest. 29, 318-324.

Klokk, T.I., Kilander, A., Xi, Z., Waehre, H., Risberg, B., Danielsen, H.E., and Saatcioglu, F. (2007). Kallikrein 4 is a proliferative factor that is overexpressed in prostate cancer. Cancer Res. 67, 5221-5230.

Kojima, K., Fujita, Y., Nozawa, Y., Deguchi, T., and Ito, M. (2010). MiR-34a attenuates paclitaxel-resistance of hormone-refractory prostate cancer PC3 cells through direct and indirect mechanisms. Prostate 70, 1501-1512.

Kong, D., Li, Y., Wang, Z., Banerjee, S., Ahmad, A., Kim, H.R., and Sarkar, F.H. (2009). miR-200 regulates PDGF-D-mediated epithelial-mesenchymal transition, adhesion, and invasion of prostate cancer cells. Stem Cells 27, 1712-1721.

Korkmaz, K.S., Korkmaz, C.G., Pretlow, T.G., and Saatcioglu, F. (2001). Distinctly different gene structure of KLK4/KLK-L1/ prostase/ARM1 compared with other members of the kallikrein family: intracellular localization, alternative cDNA forms, and regulation by multiple hormones. DNA Cell Biol. 20, 435-445.

Lee, C.Y., Rennie, P.S., and Jia, W.W. (2009a). MicroRNA regulation of oncolytic herpes simplex virus-1 for selective killing of prostate cancer cells. Clin. Cancer Res. 15, 5126-5135.

Lee, K.H., Chen, Y.L., Yeh, S.D., Hsiao, M., Lin, J.T., Goan, Y.G., and Lu, P.J. (2009b). MicroRNA-330 acts as tumor suppressor and induces apoptosis of prostate cancer cells through E2F1-mediated suppression of Akt phosphorylation. Oncogene 28, 33603370.

Lewis, B.P., Burge, C.B., and Bartel, D.P. (2005). Conserved seed pairing, often flanked by adenosines, indicates that thousands of human genes are microRNA targets. Cell 120, 15-20.

Li, M., Lee, K.F., Lu, Y., Clarke, I., Shih, D., Eberhart, C., Collins, V.P., Van, M.T., Picard, D., Zhou, L., et al. (2009a). Frequent amplification of a chr19q13.41 microRNA polycistron in aggressive primitive neuroectodermal brain tumors. Cancer Cell 16, 533-546.

Li, T., Li, D., Sha, J., Sun, P., and Huang, Y. (2009b). MicroRNA-21 directly targets MARCKS and promotes apoptosis resistance and invasion in prostate cancer cells. Biochem. Biophys. Res. Commun. 383, 280-285.

Liu, C., Kelnar, K., Liu, B., Chen, X., Calhoun-Davis, T., Li, H., Patrawala, L., Yan, H., Jeter, C., Honorio, S., et al. (2011a). The microRNA miR-34a inhibits prostate cancer stem cells and metastasis by directly repressing CD44. Nat. Med. 17, 211-215.

Liu, L.Z., Li, C., Chen, Q., Jing, Y., Carpenter, R., Jiang, Y., Kung, H.F., Lai, L., and Jiang, B.H. (2011b). MiR-21 induced angiogenesis through AKT and ERK activation and HIF-1alpha expression. PLoS One 6, e19139. 
Long, Q., Johnson, B.A., Osunkoya, A.O., Lai, Y.H., Zhou, W., Abramovitz, M., Xia, M., Bouzyk, M.B., Nam, R.K., Sugar, L., et al. (2011). Protein-coding and microRNA biomarkers of recurrence of prostate cancer following radical prostatectomy. Am. J. Pathol. 179, 46-54.

Luo, L.Y. and Diamandis, E.P. (2000). Down-regulation of the normal epithelial cell-specific 1 (NES1) gene is associated with unfavourable outcome of prostate cancer. Clin. Biochem. 33, 237.

Metias, S.M., Lianidou, E., and Yousef, G.M. (2009). MicroRNAs in clinical oncology: at the crossroads between promises and problems. J. Clin. Pathol. 62, 771-776.

Musumeci, M., Coppola, V., Addario, A., Patrizii, M., MaugeriSacca, M., Memeo, L., Colarossi, C., Francescangeli, F., Biffoni, M., Collura, D., et al. (2011). Control of tumor and microenvironment cross-talk by miR-15a and miR-16 in prostate cancer. Oncogene 30, 4231-4242.

Noonan, E.J., Place, R.F., Pookot, D., Basak, S., Whitson, J.M., Hirata, H., Giardina, C., and Dahiya, R. (2009). miR-449a targets HDAC-1 and induces growth arrest in prostate cancer. Oncogene 28, 1714-1724.

Noonan, E.J., Place, R.F., Basak, S., Pookot, D., and Li, L.C. (2010). miR-449a causes Rb-dependent cell cycle arrest and senescence in prostate cancer cells. Oncotarget 1, 349-358.

Nupponen, N.N. and Visakorpi, T. (2000). Molecular cytogenetics of prostate cancer. Microsc. Res. Tech. 51, 456-463.

Obiezu, C.V., Soosaipillai, A., Jung, K., Stephan, C., Scorilas, A., Howarth, D.H., and Diamandis, E.P. (2002). Detection of human kallikrein 4 in healthy and cancerous prostatic tissues by immunofluorometry and immunohistochemistry. Clin. Chem. 48, 1232-1240.

Peng, X., Guo, W., Liu, T., Wang, X., Tu, X., Xiong, D., Chen, S., Lai, Y., Du, H., Chen, et al. (2011). Identification of miRs-143 and -145 that is associated with bone metastasis of prostate cancer and involved in the regulation of EMT. PLoS One 6, e20341.

Qin, W., Zhao, B., Shi, Y., Yao, C., Jin, L., and Jin, Y. (2009). BMPRII is a direct target of miR-21. Acta Biochim. Biophys. Sin.(Shanghai) 41, 618-623.

Qin, W., Shi, Y., Zhao, B., Yao, C., Jin, L., Ma, J., and Jin, Y. (2010). miR-24 regulates apoptosis by targeting the open reading frame (ORF) region of FAF1 in cancer cells. PLoS One 5, e9429.

Recker, F., Kwiatkowski, M.K., Piironen, T., Pettersson, K., Huber, A., Lummen, G., and Tscholl, R. (2000). Human glandular kallikrein as a tool to improve discrimination of poorly differentiated and non-organ-confined prostate cancer compared with prostate-specific antigen. Urology 55, 481-485.

Ribas, J. and Lupold, S.E. (2010). The transcriptional regulation of miR-21, its multiple transcripts, and their implication in prostate cancer. Cell Cycle 9, 923-929.

Ribas, J., Ni, X., Haffner, M., Wentzel, E.A., Salmasi, A.H., Chowdhury, W.H., Kudrolli, T.A., Yegnasubramanian, S., Luo, J., Rodriguez, R., et al. (2009). miR-21: an androgen receptorregulated microRNA that promotes hormone-dependent and hormone-independent prostate cancer growth. Cancer Res. 69, 7165-7169.

Sarkar, F.H., Li, Y., Wang, Z., and Kong, D. (2010). Novel targets for prostate cancer chemoprevention. Endocr. Relat. Cancer 17, R195-R212.

Schaefer, A., Jung, M., Mollenkopf, H.J., Wagner, I., Stephan, C., Jentzmik, F., Miller, K., Lein, M., Kristiansen, G., and Jung, K. (2010a). Diagnostic and prognostic implications of microRNA profiling in prostate carcinoma. Int. J. Cancer 126, 1166-1176.
Schaefer, A., Stephan, C., Busch, J., Yousef, G.M., and Jung, K. (2010b). Diagnostic, prognostic and therapeutic implications of microRNAs in urologic tumors. Nat. Rev. Urol. 7, 286-297.

Schramedei, K., Morbt, N., Pfeifer, G., Lauter, J., Rosolowski, M., Tomm, J.M., von, B.M., Horn, F., and Brocke-Heidrich, K. (2011). MicroRNA-21 targets tumor suppressor genes ANP32A and SMARCA4. Oncogene 30, 2975-2985.

Seiz, L., Kotzsch, M., Grebenchtchikov, N.I., Geurts-Moespot, A.J., Fuessel, S., Goettig, P., Gkazepis, A., Wirth, M.P., Schmitt, M., Lossnitzer, A., et al. (2010). Polyclonal antibodies against kallikrein-related peptidase 4 (KLK4): immunohistochemical assessment of KLK4 expression in healthy tissues and prostate cancer. Biol. Chem. 391, 391-401.

Shi, G.H., Ye, D.W., Yao, X.D., Zhang, S.L., Dai, B., Zhang, H.L., Shen, Y.J., Zhu, Y., Zhu, Y.P., Xiao, W.J., et al. (2010). Involvement of microRNA-21 in mediating chemo-resistance to docetaxel in androgen-independent prostate cancer PC3 cells. Acta Pharmacol. Sin. 31, 867-873.

Shi, X.B., Xue, L., Ma, A.H., Tepper, C.G., Kung, H.J., and White, R.W. (2011). miR-125b promotes growth of prostate cancer xenograft tumor through targeting pro-apoptotic genes. Prostate $71,538-549$.

Sidiropoulos, M., Pampalakis, G., Sotiropoulou, G., Katsaros, D., and Diamandis, E.P. (2005). Downregulation of human kallikrein 10 (KLK10/NES1) by CpG island hypermethylation in breast, ovarian and prostate cancers. Tumour Biol. 26, 324-336.

Stephan, C., Jung, K., Nakamura, T., Yousef, G.M., Kristiansen, G., and Diamandis, E.P. (2006). Serum human glandular kallikrein 2 (hK2) for distinguishing stage and grade of prostate cancer. Int. J. Urol. 13, 238-243.

Sun, D., Lee, Y.S., Malhotra, A., Kim, H.K., Matecic, M., Evans, C., Jensen, R.V., Moskaluk, C.A., and Dutta, A. (2011). miR-99 family of MicroRNAs suppresses the expression of prostate-specific antigen and prostate cancer cell proliferation. Cancer Res. 71, $1313-1324$.

Sylvestre, Y., De, G.V., Querido, E., Mukhopadhyay, U.K., Bourdeau, V., Major, F., Ferbeyre, G., and Chartrand, P. (2007). An E2F/ miR-20a autoregulatory feedback loop. J. Biol. Chem. 282, 2135-2143.

Szczyrba, J., Loprich, E., Wach, S., Jung, V., Unteregger, G., Barth, S., Grobholz, R., Wieland, W., Stohr, R., Hartmann, A., et al. (2010). The microRNA profile of prostate carcinoma obtained by deep sequencing. Mol. Cancer Res. 8, 529-538.

Veveris-Lowe, T.L., Lawrence, M.G., Collard, R.L., Bui, L., Herington, A.C., Nicol, D.L., and Clements, J.A. (2005). Kallikrein 4 (hK4) and prostate-specific antigen (PSA) are associated with the loss of E-cadherin and an epithelial-mesenchymal transition (EMT)-like effect in prostate cancer cells. Endocr. Relat. Cancer 12, 631-643.

Wang, W.X., Kyprianou, N., Wang, X., and Nelson, P.T. (2010). Dysregulation of the mitogen granulin in human cancer through the miR-15/107 microRNA gene group. Cancer Res. 70, 9137-9142.

White, N.M. and Yousef, G.M. (2010). MicroRNAs: exploring a new dimension in the pathogenesis of kidney cancer. BMC Med. 8,65 .

White, N.M. and Yousef, G.M. (2011). Translating molecular signatures of renal cell carcinoma into clinical practice. J. Urol. 186, 9-11.

White, N.M., Bui, A., Mejia-Guerrero, S., Chao, J., Soosaipillai, A., Youssef, Y., Mankaruos, M., Honey, R.J., Stewart, R., Pace, K.T., et al. (2010a). Dysregulation of kallikrein-related peptidases in renal cell carcinoma: potential targets of miRNAs. Biol. Chem. 391, 411-423. 
White, N.M., Chow, T.F., Mejia-Guerrero, S., Diamandis, M., Rofael, Y., Faragalla, H., Mankaruous, M., Gabril, M., Girgis, A., and Yousef, G.M. (2010b). Three dysregulated miRNAs control kallikrein 10 expression and cell proliferation in ovarian cancer. Br. J. Cancer 102, 1244-1253.

White, N.M., Bao, T.T., Grigull, J., Youssef, Y.M., Girgis, A., Diamandis, M., Fatoohi, E., Metias, M., Honey, R.J., Stewart, R., et al. (2011). MiRNA profiling in clear cell renal cell carcinoma: biomarker discovery and the identification of potential controls and consequences of miRNA dysregulation. J. Urol. 186, 1077-1083.

Xi, Z., Klokk, T.I., Korkmaz, K., Kurys, P., Elbi, C., Risberg, B., Danielsen, H., Loda, M., and Saatcioglu, F. (2004). Kallikrein 4 is a predominantly nuclear protein and is overexpressed in prostate cancer. Cancer Res. 64, 2365-2370.

Xiao, F., Zuo, Z., Cai, G., Kang, S., Gao, X., and Li, T. (2009). miRecords: an integrated resource for microRNA-target interactions. Nucleic Acids Res. 37, D105-D110.

Xu, B., Niu, X., Zhang, X., Tao, J., Wu, D., Wang, Z., Li, P., Zhang, W., Wu, H., Feng, N., et al. (2011). miR-143 decreases prostate cancer cells proliferation and migration and enhances their sensitivity to docetaxel through suppression of KRAS. Mol. Cell. Biochem. 350, 207-213.

Yang, C.H., Yue, J., Fan, M., and Pfeffer, L.M. (2010). IFN induces miR-21 through a signal transducer and activator of transcription 3-dependent pathway as a suppressive negative feedback on IFNinduced apoptosis. Cancer Res. 70, 8108-8116.

Yousef, G.M. (2008). microRNAs: a new frontier in kallikrein research. Biol. Chem. 389, 689-694.

Yousef, G.M., Obiezu, C.V., Luo, L.Y., Black, M.H., and Diamandis, E.P. (1999). Prostase/KLK-L1 is a new member of the human kallikrein gene family, is expressed in prostate and breast tissues, and is hormonally regulated. Cancer Res. 59, 4252-4256.

Yousef, G.M., Scorilas, A., Jung, K., Ashworth, L.K., and Diamandis, E.P. (2001). Molecular cloning of the human kallikrein 15 gene (KLK15). Up-regulation in prostate cancer. J. Biol. Chem. 276, 53-61.

Yousef, G.M., Polymeris, M.E., Yacoub, G.M., Scorilas, A., Soosaipillai, A., Popalis, C., Fracchioli, S., Katsaros, D., and Diamandis, E.P. (2003). Parallel overexpression of seven kallikrein genes in ovarian cancer. Cancer Res. 63, 2223-2227.

Zaman, M.S., Chen, Y., Deng, G., Shahryari, V., Suh, S.O., Saini, S., Majid, S., Liu, J., Khatri, G., Tanaka, Y., et al. (2010). The functional significance of microRNA-145 in prostate cancer. Br. J. Cancer 103, 256-264.

Zhang, Y., Zhang, R., and Su, B. (2009). Diversity and evolution of MicroRNA gene clusters. Sci. China C. Life Sci. 52, 261-266.

Received November 3, 2011; accepted January 11, 2012 